\title{
Genetic analysis of sinonasal adenocarcinoma phenotypes: distinct alterations of histogenetic significance
}

\author{
Sue S Yom ${ }^{1}$, Asif Rashid ${ }^{2}$, David I Rosenthal ${ }^{1}$, Danielle D Elliott ${ }^{2}$, Ehab Y Hanna ${ }^{3}$, \\ Randal S Weber ${ }^{3}$, Adel K El-Naggar ${ }^{2,3}$ \\ ${ }^{1}$ Department of Radiation Oncology, The University of Texas MD Anderson Cancer Center, Houston, TX, \\ USA; ${ }^{2}$ Department of Pathology, The University of Texas MD Anderson Cancer Center, Houston, TX, USA \\ and ${ }^{3}$ Department of Head and Neck Surgery, The University of Texas MD Anderson Cancer Center, Houston, \\ TX, USA
}

\begin{abstract}
Sinonasal adenocarcinomas, a relatively rare entity, are composed of distinctly different morphologic subtypes with variable biological behavior. To investigate the genetic events associated with their development and clinicopathologic features, we analyzed the alterations in K-ras, APC, $\beta$-catenin, hMLH1 and hMSH2 and p53 genes expression in a cohort of 15 primary tumors comprising the two main sinonasal adenocarcinoma subtypes (enteric and seromucinous). The patients consisted of 13 men and two women, who ranged in age from 50 to 87 years. Tumors were predominantly located in the ethmoid sinus. Eight tumors were Enteric-type, and seven were seromucinous type. Nine patients were smokers and four were nonsmokers; and no information was available on two patients. Two of the eight enteric-type, had K-ras mutation at codons $12 \mathrm{~A}$ and 12B, and one showed microsatellite instability at BAT-25. Two patients with enteric-type tumors had a history of wood-dust exposure, and one had a K-ras mutation at 12A codon as well as p53 overexpression. No patients with the seromucinous type had any genetic abnormalities, except for overexpression of p53 in two tumors. Our results show that (1) a subset of enteric-type sinonasal adenocarcinoma shares certain genetic alterations with colonic adenocarcinomas, (2) the seromucinous-type sinonasal adenocarcinoma lacks alterations and may develop through a different pathway, (3) high p53 expression is associated with aggressive tumor features in both subtypes and (4) the enteric-type runs a more malignant course than the seromucinous counterpart.

Modern Pathology (2005) 18, 315-319, advance online publication, 15 October 2004; doi:10.1038/modpathol.3800315
\end{abstract}

Keywords: sinonasal adenocarcinoma; enteric-type adenocarcinoma; seromucinous carcinoma; molecular analysis

Primary sinonasal adenocarcinomas (SNACs), excluding those of salivary origin, are uncommon and represent approximately $10-20 \%$ of malignant neoplasms at these locations. Despite their common origin from an ectodermally derived respiratory mucosa, they manifest two distinct phenotypic categories, including the enteric and the seromucinous adenocarcinomas. ${ }^{1,2}$ The underlying mechanism for their histopathologic diversity is unknown. Our group has recently shown that the respiratory epithelium undergoes intestinal epithelial metaplasia (Figure 2a) prior to the development of the

Correspondence: Dr AK El-Naggar, MD, PhD, Department of Pathology, University of Texas MD Anderson Cancer Center, 1515 Holcombe Blvd., Houston, TX 77030, USA.

E-mail: anaggar@mdanderson.org

Received 16 August 2004; revised and accepted 26 August 2004; published online 15 October 2004 enteric-form of sinonasal adenocarcinoma, supporting histogenetic resemblance to primary colonic carcinoma. ${ }^{3}$ We, therefore, hypothesize that the enteric-type shares common genetic alterations with primary colonic adenocarcinoma and differs from the seromucinous-type.

Previous investigations have shown that certain genetic alterations at the adenomatous polyposis coli (APC), $\beta$-catenin and $K$-ras genes, and mutations and/or deletions of the p53 suppressor gene characterize colorectal tumorigenesis. ${ }^{4-14}$ Also, microsatellite instability (MSI) caused by alterations in nucleotide mismatch repair genes, including hMSH2, hMLH1, PMS1, PMS2 and GTBP, has been reported to be associated with a subset of these tumors. ${ }^{10-12}$ Molecular studies of SNAC are rare and limited to the intestinal type and have reported contradictory results. Wu et $a l^{13}$ in a study of the enteric form, reported a lack of point mutations in 

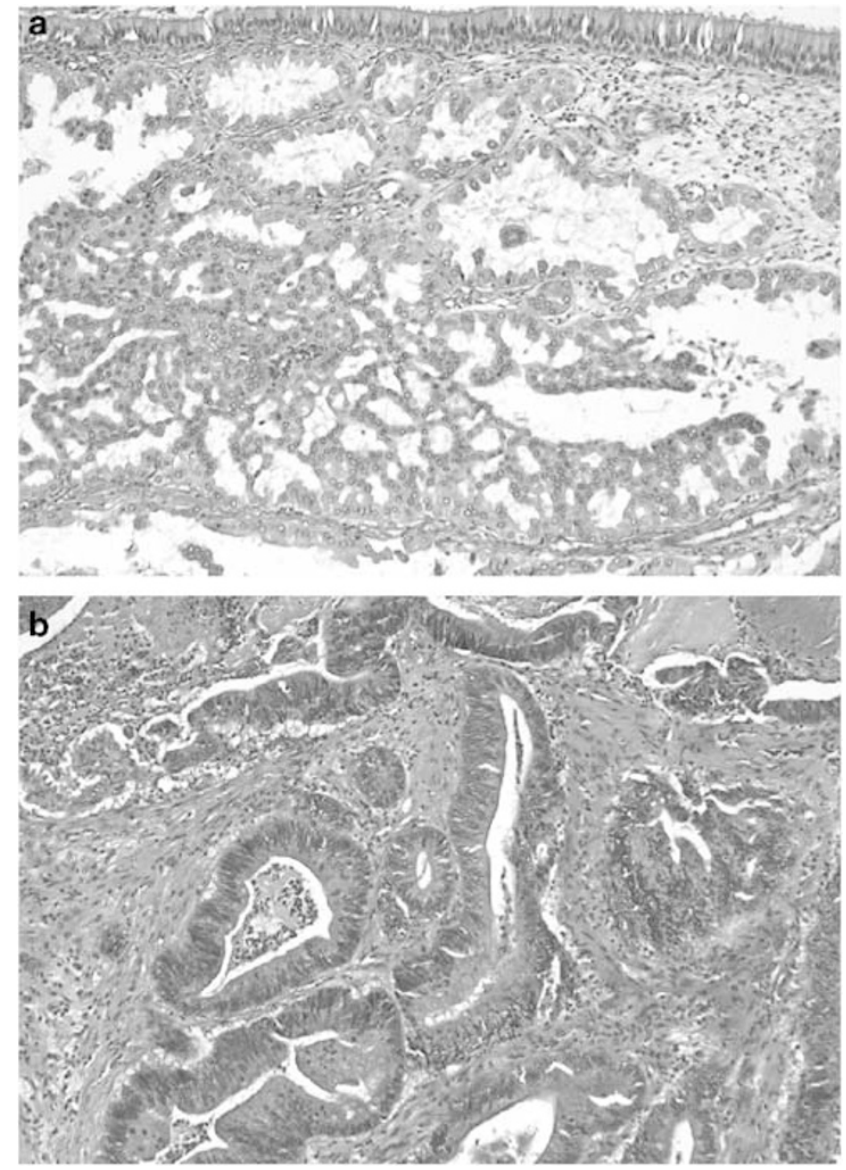

Figure 1 Photomicrographs of seromucinous (a) and intestinaltype (b).

the first or second exon of the K-ras oncogene, and only two p53 mutations in 11 specimens. However, a recent study by Saber et $a l^{14}$ found four K-ras mutations at codons 12 or 13 in 28 patients with sinonasal adenocarcinoma, while only one of 13 enteric-type tumors had K-ras mutation.

To examine the molecular events associated with the phenotypic diversity of SNAC, we performed molecular and immunohistochemical analysis on 15 primary tumors of both types.

\section{Materials and methods}

\section{Specimens}

The surgical pathology database of MD Anderson Cancer Center from 1995 to 2003 was searched and 16 patients were identified who had sinonasal adenocarcinomas with available tissue blocks from curative resection or tumor biopsy. All slides were reviewed to confirm the original diagnosis and to select blocks for this study. Histologic classification was based on criteria of Kleinsasser ${ }^{15}$ and Batsakis et al. ${ }^{16}$ Histological slides were reviewed by two pathologists operating independently of clinical information. Patient information was extracted from medical a
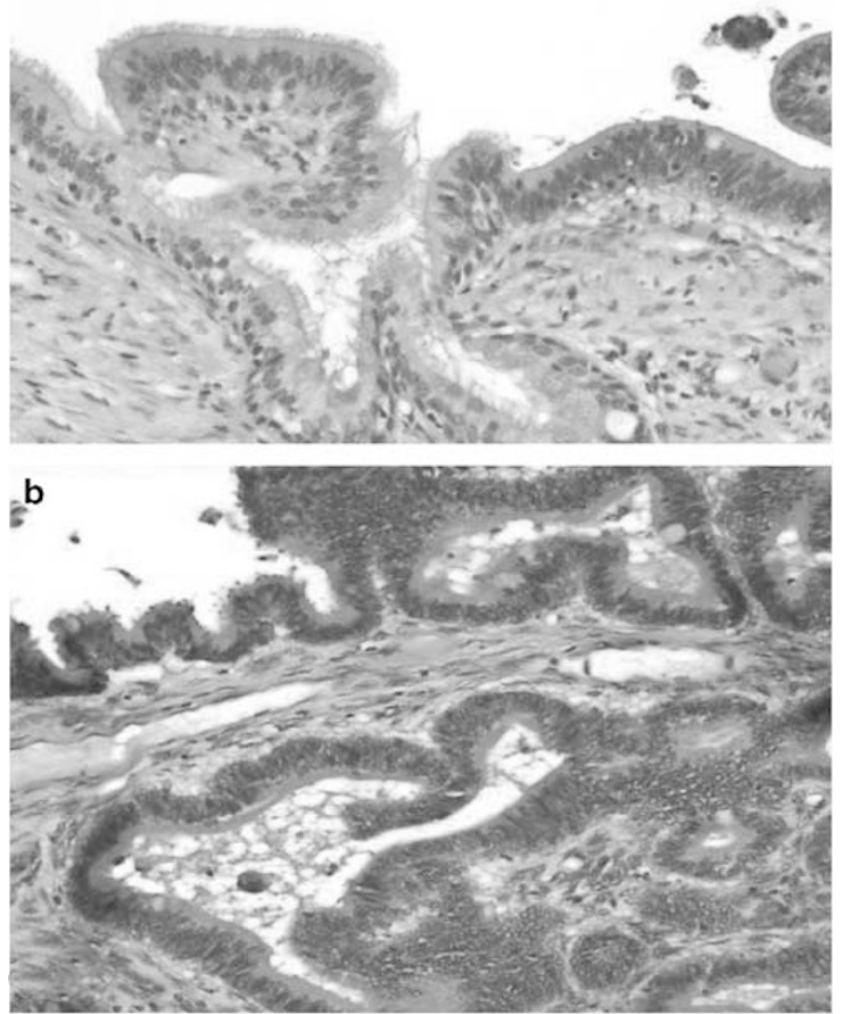

Figure 2 Intestinal metaplasia of the respiratory epithelium (a) and underlying intestinal-type sinonasal adenocarcinoma (b).

records. Records were reviewed for age, sex, race, history of exposure to dust or smoking, site and stage of tumor, type of treatment, and follow-up status.

\section{DNA Extraction}

DNA was prepared from tissue specimens stored in paraffin-embedded, $4 \%$ formalin-fixed blocks. In a few cases for which this isolation technique was insufficient, genomic DNA was extracted by microdissection of hematoxylin- and eosin-stained slides without coverslip. A 27 1/2-gauge needle was used under low magnification $(\times 4)$ in selected areas where the neoplastic cellularity was $>50 \%$. Genomic DNA was extracted from the microdissected tissue, as described previously. ${ }^{17}$ Briefly, three 5- $\mu \mathrm{m}$ thick sections were extracted twice in $1000 \mu \mathrm{l}$ of xylene for $30 \mathrm{~min}$ and twice in $1000 \mu \mathrm{l}$ of $100 \%$ ethanol for 3-5 min. The solid residue was dried by evaporation, after which $75 \mu \mathrm{l}$ of lysis buffer was added $(0.25 \%$ Tween-20, TE9, $2 \mathrm{mg} / \mathrm{ml}$ Proteinase $\mathrm{K})$. The mixture was incubated overnight at $56^{\circ} \mathrm{C}$. The Proteinase $\mathrm{K}$ was deactivated for $10 \mathrm{~min}$ at $100^{\circ} \mathrm{C}$. The condensate was separated from the insoluble material by centrifugation. 


\section{Molecules Analyses}

DNA could not be isolated in one patient with enteric-type. Therefore, seven enteric-type and seven nonseromucinous tumors were analyzed.

Analysis of K-ras, APC, and $\beta$-catenin gene mutation was carried out as previously described. ${ }^{5}$ Immunohistochemistry for microsatellite analysis of markers hMLH1 and hMSH2 was carried out according to the method of Alexander et al. ${ }^{18}$

Immunohistochemistry for p53 protein was also performed according to previously published method. ${ }^{19}$ Overexpression of p53 protein was considered to be present when more than $50 \%$ of the nuclei of tumor cells were strongly stained.

\section{Results}

\section{Clinical Data}

The clinicopathologic findings of all sinonasal adenocarcinoma cases are summarized in Table 1. Histopathologically, seven tumors were seromucinous-type (Figure 1a) and eight were enteric-type (Figures $1 \mathrm{~b}$ and 2b). All of the seromucinous adenocarcinomas were of the low-grade category. Six of the eight enteric-type carcinomas were moderately differentiated villo-tubular adenocarcinomas and two were well-differentiated adenocarcinoma with mucinous component. Of the 15 primary tumors, 13 occurred in men and two in women. The ages ranged from 50 to 87 years, with a mean of 67 years. Ten primary tumors originated in the ethmoid sinus, one in the maxillary sinus and two in the nasal cavity; and the primary site was unknown for two cases. Six of eight patients with enteric-type carcinomas and three of seven with seromucinous form had a history of smoking. Two of the eight patients with the enteric-type carcinoma had a history of wood-dust exposure and were also smokers. Initial surgical treatment included five craniofacial resections, four medial maxillectomies, two endoscopic resections, one partial maxillectomy, one transpalatal resection, and two unknown.

\section{Genetic Alterations}

Table 2 presents the molecular findings of sinonasal adenocarcinoma. Two K-ras mutations at codon 12 were identified in two of seven enteric-type carcinomas $(29 \%)$ but in none of the seromucinous carcinomas (Figure 3). One of these tumors manifested associated p53 overexpression and MSI $(14 \%)$. Two of seven (29\%) seromucinous-type tumors had p53 overexpression, and none had any genetic alterations at the genes tested. No mutation at the APC or $\beta$-catenin genes was present in any tumor of either type. Alteration in hMLH1 expression was identified in two moderately differentiated enteric-type carcinomas, and one also had alteration of hMSH2. None of the seromucinous cancers showed MSI.

\section{Discussion}

Our results show K-ras mutations in 29\% of the enteric-type carcinoma tumors and in none of the seromucinous-subtype. A similar incidence of mutations at this gene has previously been reported in studies of primary colorectal carcinomas. ${ }^{14}$ This is further underscored by the detection of an MSI, a feature commonly present in a subset of primary enteric adenocarcinomas, in one of the enteric-type with K-ras mutation. Because of the phenotypic similarities between primary colonic and enterictype sinonasal adenocarcinomas the presence of Kras mutations in both indicates an early association

Table 1 Demographic, clinicopathologic and follow-up data of patients with sinonasal adenocarcinomas

\begin{tabular}{|c|c|c|c|c|c|c|c|c|c|c|c|}
\hline Tyре & Age & Sex & Race & Risk factors & Site & P. Surgery & Stage & Margins & $X R T$ & CHX & Follow-up \\
\hline \multicolumn{12}{|c|}{ Enteric-type } \\
\hline 1 & 73 & $\mathrm{M}$ & White & Smoking (20 py) & ES & CFR & 4 & Negative & N/A & $\mathrm{N} / \mathrm{A}$ & N/A \\
\hline 2 & 67 & $\mathrm{M}$ & White & None & ES & ER & 2 & Positive & Yes & Yes & LR \\
\hline 3 & 68 & $\mathrm{M}$ & White & Smoking ( 20 py), chemicals, asbestos & ES & MM & 2 & Negative & Yes & No & NED \\
\hline 4 & 53 & $\mathrm{M}$ & Black & Wood dust; smoking (20 py) & ES & CFR & 4 & Negative & No & No & NED \\
\hline 5 & 76 & $\mathrm{M}$ & White & Smoking ( 20 py) & ES & ER & 2 & Positive & Yes & No & LR \\
\hline 6 & 71 & $\mathrm{~F}$ & N/A & N/A & N/A & N/A & N/A & N/A & N/A & N/A & N/A \\
\hline 7 & 67 & $\mathrm{M}$ & White & Wood dust; smoking (175 pv) & ES & PM & 2 & Positive & Yes & No & LR \\
\hline 8 & 67 & $\mathrm{M}$ & White & Smoking (42 py) & ES & CFR & 4 & Negative & Yes & No & NED \\
\hline \multicolumn{12}{|c|}{ Nonenteric } \\
\hline 9 & 71 & $\mathrm{M}$ & White & None & NC & ER & 2 & Negative & Yes & No & NED \\
\hline 10 & 68 & $\mathrm{~F}$ & $\mathrm{~N} / \mathrm{A}$ & $\mathrm{N} / \mathrm{A}$ & N/A & N/A & N/A & $\mathrm{N} / \mathrm{A}$ & N/A & N/A & N/A \\
\hline 11 & 69 & $\mathrm{M}$ & White & Smoking (90 py) & ES & MM & 2 & Negative & Yes & No & NED \\
\hline 12 & 67 & $\mathrm{M}$ & Asian & Smoking (47 py) & $\mathrm{NC}$ & MM & 2 & Positive & Yes & No & NED \\
\hline 13 & 50 & $\mathrm{M}$ & White & None & ES & CFR & 2 & Negative & Yes & No & NED \\
\hline 14 & 51 & $\mathrm{M}$ & White & None & MS & CFR & 4 & Negative & Yes & Yes & DOD \\
\hline 15 & 87 & $\mathrm{M}$ & Hispanic & Smoking (10 py) & ES & MM & 2 & Negative & N/A & N/A & NED \\
\hline
\end{tabular}

N/A, not available; PY, pack-years; CFR, craniofacial resection; ER, endoscopic resection; MM, medial maxillectomy; LR, local recurrence; NED, no evidence of disease; DOD, died of disease; PM, partial maxillectomy; ES, ethmoid sinus; NC, nasal cavity; MS, maxillary sinus. 
Table 2 Molecular analysis of sinonasal adenocarcinomas

\begin{tabular}{|c|c|c|c|c|c|c|c|c|}
\hline Type \# & $K$-ras & $A P C$ & $\beta$-Catenin & p53 & $h M L H 1$ & hMSH2 & Bat 25 & Bat 26 \\
\hline \multicolumn{9}{|l|}{ Enteric } \\
\hline 1 & $\bullet$ & 0 & $\bigcirc$ & 0 & $\bigcirc$ & 0 & 0 & $\bigcirc$ \\
\hline 2 & 0 & 0 & 0 & 0 & 0 & 0 & 0 & 0 \\
\hline 3 & 0 & 0 & 0 & 0 & 0 & 0 & 0 & 0 \\
\hline 4 & 0 & 0 & O & 0 & O & 0 & 0 & 0 \\
\hline 5 & O & 0 & O & ○ & O & O & $\bigcirc$ & 0 \\
\hline 6 & 0 & 0 & 0 & 0 & 0 & 0 & 0 & 0 \\
\hline 7 & 0 & 0 & 0 & 0 & 0 & 0 & 0 & 0 \\
\hline 8 & N/A & N/A & N/A & N/A & N/A & N/A & N/A & N/A \\
\hline \multicolumn{9}{|c|}{ Non-enteric } \\
\hline 9 & 0 & $\bigcirc$ & O & 0 & $\bigcirc$ & 0 & $\bigcirc$ & 0 \\
\hline 10 & O & $\bigcirc$ & O & 0 & O & 0 & $\bigcirc$ & 0 \\
\hline 11 & 0 & N/A & $\bigcirc$ & 0 & 0 & 0 & 0 & 0 \\
\hline 12 & 0 & $\bigcirc$ & $\bigcirc$ & 0 & 0 & 0 & 0 & 0 \\
\hline 13 & 0 & 0 & 0 & 0 & 0 & 0 & 0 & 0 \\
\hline 14 & O & $\bigcirc$ & O & 0 & O & O & N/A & 0 \\
\hline 15 & O & $\bigcirc$ & O & $\bigcirc$ & O & $\bigcirc$ & $\bigcirc$ & O \\
\hline
\end{tabular}

๑, alteration; $\bigcirc$, no alteration; N/A, not assessed.

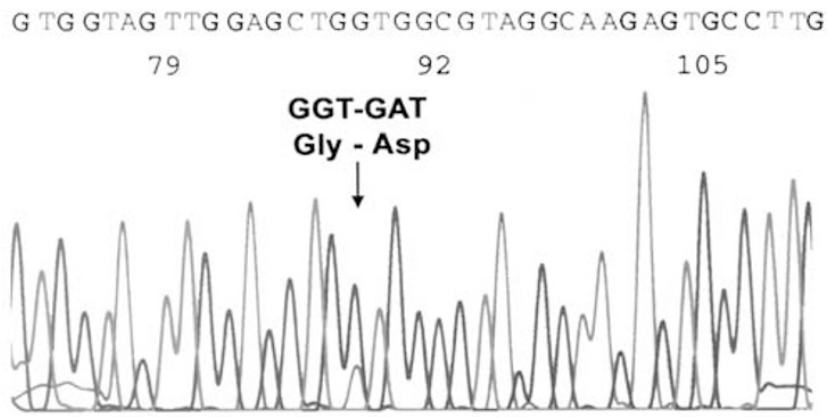

G G TAG TTG G AGC T NG TGG CG TAGGCAAGAG TGC C TTG A

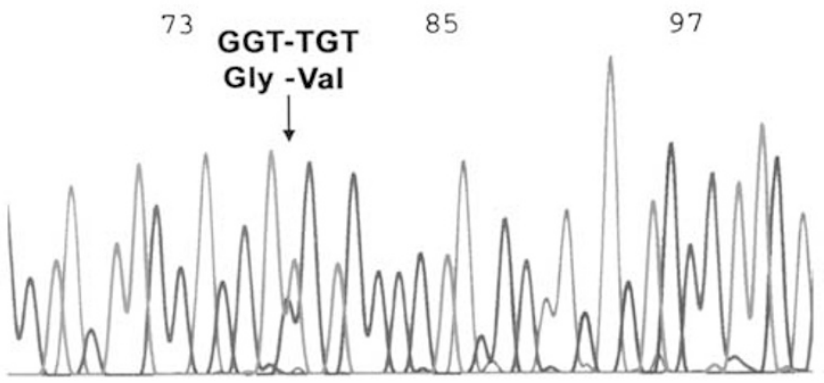

Figure $3 \mathrm{G} \rightarrow$ A transition mutation of the Ras gene in intestinaltype sinonasal adenocarcinoma.

with their development, regardless of the site of origin. In addition evidence that intestinal metaplasia of the sinonasal mucosa precede to the development of the enteric-type lends further credence to this hypothesis. ${ }^{3}$

The lack of other genetic features typically reported in primary colonic adenocarcinoma in our enteric-type tumors, however, suggest that specific events related to their site of origin underlie these differences. We contend that subsequent genetic events, especially in the former, are most likely acquired during the progression as a result of loco- regional and other epidemiological factors. ${ }^{20-27}$ These results, along with the lack of any alteration in the seromucinous subtype, support a distinct pathway for the evolution and progression of the intestinal and primary colonic adenocarcinomas.

In our cohort, only three high-grade tumors overexpressed p53, one of which was enteric in type and also had a K-ras mutation. Two patients with these tumors developed local recurrence or metastasis. Studies of colonic adenocarcinoma and a recent study of sinonasal adenocarcinoma have reported a high incidence of p53 abnormalities. ${ }^{28,29}$ Recent studies of p53 in enteric-type adenocarcinoma have associated this finding with sawdust exposure. ${ }^{13,28,29}$ Similarly an association between dust exposure and epigenetic alterations at the CpG island of certain genes in these tumors has been reported. ${ }^{28}$ The underlying factors for the differences between our findings and those of other investigators can be attributed to epidemiologic and/or patient populations variables. Only two patients in our cohort had a history of wood-dust exposure, and it is conceivable that sawdust contributes only to the development of a subset of the enteric-form tumors and that an alternative pathway may play a role in tumors' developing in nondust-exposed patients.

Our findings also suggest that patients with dust exposure develop via a different pathway than those without such history, and that the enteric-form are different molecularly from seromucinous adenocarcinoma. In our cohort, patients with the enteric-form had a more aggressive clinical course, than those with the seromucinous-type. However, because of the small number of tumors examined further studies are needed to determine the effect of tumor differentiation on the clinical behavior of these tumors. Sinonasal adenocarcinomas are generally unresponsive to medical management modalities and definitive surgical excision which achieves 
local control rates of $50 \%$ is the primary treatment of choice. ${ }^{30}$ Recently, however, the addition of postoperative radiation following surgical resection have increased the 5-year local control rate to $59 \% .^{31}$ Since our patient outcomes are consistent with these findings, we, recommend that patients with the enteric-type be treated with surgery and adjuvant radiation therapy.

\section{Acknowledgements}

This work was supported in part by the Kenneth $\mathrm{D}$ Müller Professorship and a National Cancer Institute Specialized Program of Research Excellence grant in head and neck cancer.

\section{References}

1 Batsakis JG, Rice DH, Solomon AR. The pathology of head and neck tumors: squamous and mucous-gland carcinomas of the nasal cavity, paranasal sinuses, and larynx, part 6. Head Neck Surg 1980;2:497-508.

2 Franquemont DW, Fechner RE, Mills SE. Histologic classification of sinonasal-intestinal type adenocarcinoma. Am J Surg Pathol 1991;15:368-375.

3 Choi H-R, Sturgis EM, Rashid A, et al. Sinonasal adenocarcinoma: evidence for histogenetic divergence of the Enteric and nonenteric phenotypes. Hum Path 2003;34:1101-1107.

4 Vogelstein B, Fearon ER, Hamilton SR, et al. Genetic alterations during colorectal-tumor development. N Engl J Med 1988;319:525-532.

5 Lee JH, Abraham SC, Kim HS, et al. Inverse relationship between APC gene mutation in gastric adenomas and development of adenocarcinoma. Am J Pathol 2002;161:611-618.

6 Kim IJ, Kang HC, Park JH, et al. Determination of tumor aggressiveness in colorectal cancer by K-ras-2 analysis. Arch Surg 1993;128:526-531.

7 Finkelstein SD, Sayegh R, Bakker A, et al. Prediction of biologic aggressiveness in colorectal cancer by p53/Kras-2 topographic genotyping. Mol Diagn 1996;1:5-28.

8 Cunningham J, Lust JA, Schaid DJ, et al. Expression of p53 and 17p allelic loss in colorectal carcinoma. Cancer Res 1992;52:1974-1980.

9 Ohue M, Tomita N, Monden $\mathrm{T}$, et al. A frequent alteration of p53 gene in carcinoma in adenoma of colon. Cancer Res 1994;54:4798-4804.

10 Kim IJ, Kang HC, Park JH, et al. Development and applications of a beta-catenin oligonucleotide microarray: beta-catenin mutations are dominantly found in the proximal colon cancers with microsatellite instability. Clin Cancer Res 2003;9:2920-2925.

11 Fukushima H, Yamamoto H, Itoh F, et al. Frequent alterations of the beta-catenin and TCF-4 genes, but not of the APC gene, in colon cancers with high-frequency microsatellite instability. J Exp Clin Cancer Res 2001;20:553-559.

12 Fujiwara T, Stolker JM, Watanable T, et al. Accumulated clonal genetic alterations in familial and sporadic colorectal carcinomas with widespread instability in microsatellite sequences. Am J Pathol 1998;153:1063-1078.

$13 \mathrm{Wu}$ TT, Barnes L, Bakker A, et al. K-ras-2 and p53 genotyping of intestinal-type adenocarcinoma of the nasal cavity and paranasal sinuses. Mod Pathol 1996;9:199-204.

14 Saber AT, Nielsen LR, Dictor M, et al. K-ras mutations in sinonasal adenocarcinomas in patients occupationally exposed to wood or leather dust. Cancer Lett 1998; 126:59-65.

15 Kleinsasser O. Terminal tubulus adenocarcinoma of the nasal seromucous glands. A specific entity. Arch Otorhinolaryngol 1985;241:183-193.

16 Batsakis JG, Mackay B, Ordonez NG. Enteric-type adenocarcinoma of the nasal cavity. An electron microscopic and immunocytochemical study. Cancer 1984;54:855-860.

17 Goelz SE, Hamilton SR, Vogelstein B. Purification of DNA from formaldehyde fixed and paraffin embedded human tissue. Biochem Biophys Res Commun 1985; 130:118-126.

18 Alexander J, Watanable T, Wu TT, et al. Histopathological identification of colon cancer with microsatellite instability. Am J Pathol 2001;158:527-535.

19 Baas IO, Mulder J-WR, Offerhaus GJA, et al. An evaluation of six antibodies for immunohistochemistry of mutant p53 gene product in archival colorectal neoplasms. J Pathol 1994;172:5-12.

20 Cecchi F, Buiatti E, Kriebel D, et al. Adenocarcinoma of the nose and paranasal sinuses in shoemakers and woodworkers in the province of Florence, Italy (196377). Br J Ind Med 1980;37:222-225.

21 Kleinsasser O, Schroeder HG. Adenocarcinomas of the inner nose after exposure to wood dust. Morphological findings and relationships between histopathology and clinical behavior in 79 cases. Arch Otorhinolaryngol 1988;245:1-5.

22 Wills JH. Nasal carcinoma in woodworkers: a review. J Occup Med 1982;24:526-530.

23 Klintenberg C, Olofsson J, Hellquist H, et al. Adenocarcinoma of the ethmoid sinuses. A review of 28 cases with special reference to wood dust exposure. Cancer 1984;54:482-488.

24 Hayes RB, Gerin M, Raatgever JW, et al. Wood-related occupations, wood dust exposure, and sinonasal cancer. Am J Epidemiol 1986;124:569-577.

25 Leclerc A, Martinez Cortes M, et al. Sinonasal cancer and wood dust exposure: results from a case-control study. Am J Epidemiol 1994;140:340-349.

26 Van den Oever R. Occupational exposure to dust and sinonasal cancer. An analysis of 386 cases reported to the N.C.C.S.F. Cancer Registry. Acta Otorhinolaryngol Belg 1996;50:19-24.

27 Lopez JI, Nevado M, Eizaguirre B, et al. Intestinal-type adenocarcinoma of the nasal cavity and paranasal sinuses. A clinicopathologic study of 6 cases. Tumori 1990;76:250-254.

28 Perrone F, Oggionni M, Birindelli S, et al. TP53, p14ARF, p16INK4a and H-ras gene molecular analysis in intestinal-type adenocarcinoma of the nasal cavity and paranasal sinuses. Int J Cancer 2003;105:196-203.

29 Bashir AA, Robinson RA, Benda JA, et al. Sinonasal adenocarcinoma: immunohistochemical marking and expression of oncoproteins. Head Neck 2003;25:763-771.

30 Perez P, Dominguez O, Gonzalez S, et al. Ras gene mutations in ethmoid sinus adenocarcinoma: prognostic implications. Cancer 1999;86:255-264.

31 Claus F, Boterberg T, Ost P, et al. Postoperative radiotherapy for adenocarcinoma of the ethmoid sinuses: treatment results for 47 patients. Int J Radiat Oncol Biol Phys 2002;54:1089-1094. 